\title{
Electrochemical Measurements Reveal Reactive Oxygen Species in
} Stress Granules**

\author{
Keke Hu, Emily Relton, Nicolas Locker,* Nhu T. N. Phan,* and Andrew G. Ewing*
}

\begin{abstract}
Stress granules (SGs) are membrane-less organelles that assemble in the cytoplasm to organize cellular contents and promote rapid adaptation during stress. To understand how SGs contribute to physiological functions, we used electrochemical measurements to detect electroactive species in SGs. With amperometry, we discovered that reactive oxygen species (ROS) are encapsulated inside arsenite-induced SGs, and $\mathrm{H}_{2} \mathrm{O}_{2}$ is the main species. The release kinetics of $\mathrm{H}_{2} \mathrm{O}_{2}$ from single $\mathrm{SGs}$ and the number of $\mathrm{H}_{2} \mathrm{O}_{2}$ molecules were quantified. The discovery that SGs contain ROS implicates them as communicators of the cellular stresses rather than a simple endpoint. This may explain how SGs regulate cellular metabolism and stress responses. This may also help better understand their cytoprotective functions in pathological conditions associated with SGs such as neurodegenerative diseases (NDs), cancers and viral infections.
\end{abstract}

Controlling the localization of macromolecules is key for cellular functions and typically achieved by surrounding them with lipid membranes in organelles such as the nucleus. Membraneless organelles such as stress granules (SGs) are increasingly recognized as an alternative way to organize cellular components. ${ }^{[1]}$ Their formation generates high local concentrations of RNA and protein, providing an ideal platform for the regulation of fundamental processes allowing cells to rapidly adjust in response to various physiological and pathological triggers. ${ }^{[2]}$

SGs assemble to capture mRNAs and proteins during stresses including oxidative stress, heat shock, viral infection,

[*] Dr. K. Hu, Prof. N. T. N. Phan, Prof. A. G. Ewing

Department of Chemistry and Molecular Biology, University of Gothenburg

Kemivägen 10, 41296 Gothenburg (Sweden)

E-mail: nhu.phan@chem.gu.se andrewe@chem.gu.se

E. Relton, Prof. N. Locker

Faculty of Health and Medical Sciences, School of Biosciences and Medicine, University of Surrey

Guildford, Surrey GU2 7XH (UK)

E-mail: n.locker@surrey.ac.uk

[***] A previous version of this manuscript has been deposited on a preprint server (https://doi.org/10.1101/2021.03.16.435640).

(2) Supporting information and the ORCID identification number(s) for

(iD the author(s) of this article can be found under: https://doi.org/10.1002/anie.202104308.

of (c) 2021 The Authors. Angewandte Chemie International Edition published by Wiley-VCH GmbH. This is an open access article under the terms of the Creative Commons Attribution License, which permits use, distribution and reproduction in any medium, provided the original work is properly cited. proteasomal inhibition, ER (endoplasmic reticulum) stress, UV irradiation, among others. ${ }^{[3]}$ The general inhibition of protein synthesis following stress results in the dissociation of mRNAs from polysomes and their accumulation in ribonucleoprotein (RNP) complexes. ${ }^{[3]}$ This increases the concentration of cytoplasmic RNPs and their binding by aggregation of prone RNA-binding proteins (RBPs), such as G3BP1 or TIA-1, results in clustering/fusion events driven by interactions between their protein and RNA components, ultimately promoting liquid-liquid phase separation (LLPS) and SG assembly. ${ }^{[4]}$ SGs are highly dynamic, rapidly dissolving upon stress resolution to release sequestered mRNAs for future translation.

By condensing specific proteins, SGs also alter the composition and concentration of cytoplasmic proteins, affecting the course of biochemical reactions and signalling cascades. ${ }^{[5]}$ Many signalling molecules associated with diseases concentrate in SGs, suggestive of a role for SGs as signalling hubs. ${ }^{[6]}$ Furthermore, metabolic enzymes stored in SGs produce metabolites that can affect SG stability such as AdoMet or acetyl-CoA. ${ }^{[7]}$

By concentrating key signalling and cytoplasmic sensors or effectors of innate immunity SGs are at a crossroads between intracellular signalling, antiviral responses and translation control. ${ }^{[8]}$ Moreover, the dysregulation of SGs assembly is increasingly associated with neuropathologies such as amyotrophic lateral sclerosis (ALS), Parkinson's and Alzheimer's diseases. ${ }^{[9]}$ Finally, many SG proteins are aberrantly expressed in tumours and SGs are exploited by cancer cells to adapt to the adverse conditions of the tumour microenvironment. ${ }^{[10]}$ Therefore SGs are important for many normal and pathological processes. However, beyond their protein and RNA composition, very little is known about the contents of SGs, and how these impact on their functions. With clear functional connections to signalling and metabolism, we speculated that, despite the absence of membranes, SGs could act as stores for metabolites or secondary messengers.

Reactive oxygen species (ROS) are key messengers that trigger and regulate cellular signaling pathways important for a wide range of cellular processes, including proliferation and survival, antioxidant regulation, mitochondrial oxidative stress, apoptosis, aging, iron homeostasis and DNA damage response. ${ }^{[11]}$ They are produced by enzymes localized in the cytoplasm, mitochondria, peroxisome, and ER. ROS are a family of molecules that include super oxide radical $\mathrm{O}_{2}{ }^{--}$, hydroxyl radical $\mathrm{OH}^{\cdot}$ and the freely diffusible $\mathrm{H}_{2} \mathrm{O}_{2} \cdot \mathrm{H}_{2} \mathrm{O}_{2}$ is the most stable ROS and regarded as the primary example. ${ }^{[12]}$ The opposing effects of ROS in physiological processes have attracted considerable attention. They have diverse functions 
depending on the subcellular resource, location, duration and concentration of these molecules. ${ }^{[12,13]}$ Electrochemistry offers a direct and simple way to detect ROS as some are electroactive. Normally electro-oxidation is preferred as electro-reduction is susceptible to the interference of oxygen in aerobic environments. ROS detection at singlecell and subcellular levels has been successfully achieved with the development of small probes and new methodologies. ${ }^{[14]}$

Given our limited understanding of SG contents beyond proteins and RNAs, we hypothesized they might contain electroactive species. To test this, we treated human bone osteosarcoma epithelial (U2OS) cells with sodium arsenite and then isolated SGs as previously described. ${ }^{[15]}$ Electrochemical measurements were carried out with both $\mathrm{Pt}$ microelectrodes and platinized carbon fiber microelectrodes (CFME) by either electrochemical collision ("adding") or direct absorption ("dipping"). ${ }^{[16]}$ These measurements revealed the apparent presence of ROS within SGs.

The isolation protocol of SGs is shown in the SI. The "granule enriched fraction" including primarily SGs and other proteins was dispersed in homogenizing buffer. A schematic of electrochemical measurement of isolated SGs is illustrated in Figure 1 A. An applied potential of $400 \mathrm{mV}$ (vs. $\mathrm{Ag} / \mathrm{AgCl}$ ) was used as a higher potential might bring more interferences and complicate the measurement. As can be seen from the representative amperometric traces of $33-\mu \mathrm{m}$ CFMEs and $25-\mu \mathrm{m} \mathrm{Pt}$ electrodes in Figure $1 \mathrm{~B}$, current transients were observed only with the Pt electrode indicating that the detected species can be oxidized on the surface of $\mathrm{Pt}$ instead of CFME at $400 \mathrm{mV}$. These species appear to be ROS after excluding other electroactive species such as ascorbic acid, some neurotransmitters, and enzymes.

Prior to confirming the presence of ROS, we verified that these electroactive species resulted from SGs, rather than impurities or protein contaminants. To this end, SGs were further purified from the SGs-enriched fraction using immunoprecipitation, resulting in pure SG fractions. ${ }^{[15]}$ The schematic of immunoprecipitation is presented in Figure S2. The results shown in Table S1 suggest that the interference from the impurities is negligibly small, and the electroactive species are from the SGs. The Pt microelectrode was replaced with a platinized CFME in order to increase the number of active

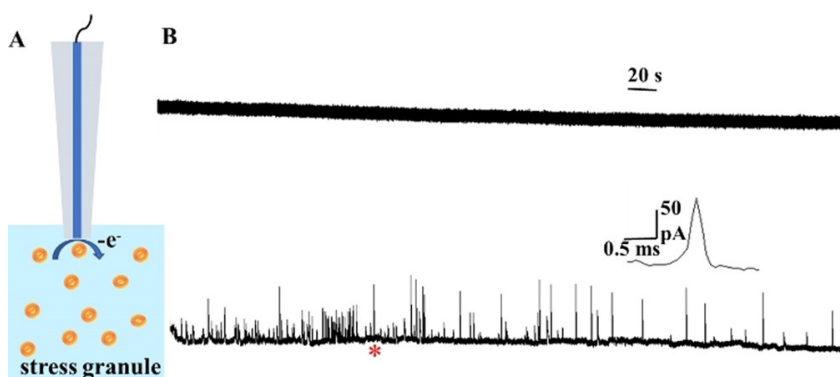

Figure 1. A) Schematic of electrochemical measurement of stress granules ( $\mathrm{SGs}$ ) isolated from $\mathrm{NaAsO}_{2}$-treated $\mathrm{U} 2 \mathrm{OS}$ cells (schematic of SGs structure is shown in Figure S1). B) Representative amperometric traces of SGs obtained from a $33 \mu \mathrm{m}$ CFME (upper trace) and a $25 \mu \mathrm{m}$ Pt electrode at $400 \mathrm{mV}$ vs. $\mathrm{Ag} / \mathrm{AgCl}$ (lower trace). The inset shows an amplification of the spike labeled with the red asterisk. sites on the electrode surface for ROS oxidation. Representative amperometric traces of $\mathrm{Pt}$ black deposition and voltammograms in $10 \mu \mathrm{M} \mathrm{H}_{2} \mathrm{O}_{2}$ before and after modification are shown in Figure S3. Platinized CFME was then dipped into the suspension of SGs for amperometric measurement. When catalase (CAT, $2.5 \mathrm{mg} \mathrm{mL}^{-1[17]}$ ), the enzyme that converts $\mathrm{H}_{2} \mathrm{O}_{2}$ to $\mathrm{O}_{2}$ and $\mathrm{H}_{2} \mathrm{O}$ is added to the solution of $\mathrm{SGs}$, the amperometric response of the same electrode is eliminated providing strong evidence that $\mathrm{H}_{2} \mathrm{O}_{2}$ is the main electroactive species in SGs. As shown in Figure 2A, spikes were observed only in the amperometric trace obtained from the suspension of SGs without CAT.

To verify that the disappearance of spikes cannot be attributed to the surface passivation of platinized CFME, another electrode was dipped into the two suspensions in a reverse order. Between these two measurements, the electrode was immersed in PBS solution for $3 \mathrm{~min}$ to remove the residues of CAT on the surface. The resultant amperometric traces are presented in Figure $2 \mathrm{~B}$ with spikes only from SGs suspension without CAT (lower trace). It should be noted that a small number of spikes were captured in some traces obtained from SGs suspension with CAT (Figure S4), indicating that in addition to $\mathrm{H}_{2} \mathrm{O}_{2}$, there might be small amount of other species in SGs. However, we conclude that the main electroactive species in arseniteinduced SGs is $\mathrm{H}_{2} \mathrm{O}_{2}$.

To quantify the release kinetics of $\mathrm{H}_{2} \mathrm{O}_{2}$ from single SGs and the number of $\mathrm{H}_{2} \mathrm{O}_{2}$ molecules, spikes with a single peak were analyzed. Based on work with vesicle impact electrochemical cytometry, we assume $\mathrm{H}_{2} \mathrm{O}_{2}$ from SGs is quantitatively oxidized. ${ }^{[18]}$ A typical spike analysis is illustrated in Figure $3 \mathrm{~A}$ with $I_{\max }$ of $22.8 \mathrm{pA}, N_{\text {molecules }}$ of $3.82 \times 10^{4}$ and

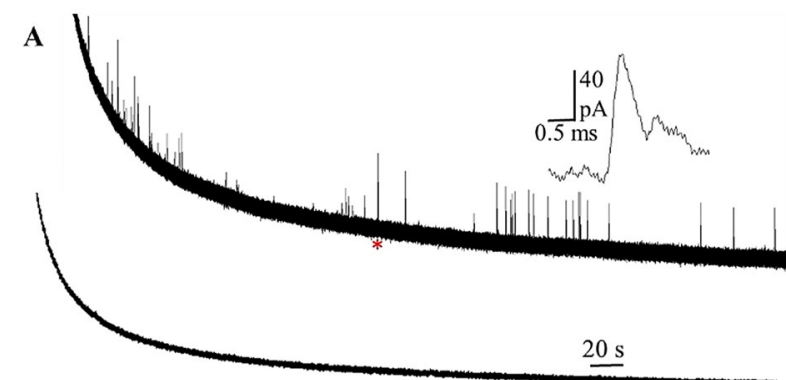

B

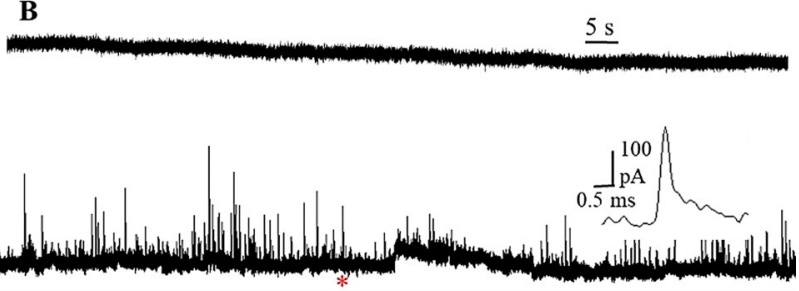

Figure 2. A) Representative amperometric traces of the same platinized CFME obtained sequentially from SGs suspensions without (upper) and with CAT (lower) at $400 \mathrm{mV}$ vs. $\mathrm{Ag} / \mathrm{AgCl}$. B) Representative amperometric traces of another platinized CFME obtained from the two suspensions in a reverse order at $400 \mathrm{mV}$ vs. $\mathrm{Ag} / \mathrm{AgCl}$. The inset shows amplification of the spikes labeled with the red asterisk. 


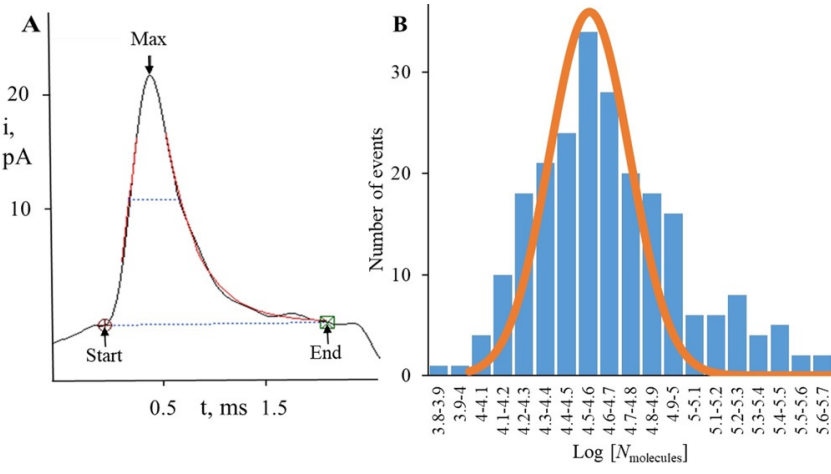

Figure 3. A) A typical spike analysis for quantification of release kinetics of $\mathrm{H}_{2} \mathrm{O}_{2}$ from single $\mathrm{SG}$ s and the number of $\mathrm{H}_{2} \mathrm{O}_{2}$ molecules. B) Distribution of log $\left[N_{\text {molecules }}\right]$. Fits were obtained from a Gaussian distribution of the data (collected from four isolations of SGs; number of events, 229).

release kinetics of $0.458 \mathrm{~ms}$. The shape of the transient matches well with theoretical curves (the red one). Statistical analysis of 229 release events shows the median for $t_{1 / 2}$ is $0.481 \mathrm{~ms}$. The dynamics for release of SGs is faster compared to both dense core vesicles and synaptic vesicles probably because of the membraneless structure or the different compositions of SGs. The distribution of $\log \left[N_{\text {molecules }}\right]$ is presented in Figure $3 \mathrm{~B}$ with a Gaussian distribution. The $N_{\text {molecules }}$ ranges from $1 \times 10^{4}$ to $1.2 \times 10^{5}$ with the median of $4.03 \times 10^{4}$.

Because cancer cells are typically enriched with ROS, we next assessed whether SGs assembled in non-cancer cells also contain ROS. To test this, we used MCF-10A non-cancer cells, which, unlike cancer cells, contain no ROS or the amount is too low to be detectable without any treatment. ${ }^{[19]}$ First, we established that arsenite stimulation of MCF-10A cells resulted in SG assembly, as detected by the accumulation of cytoplasmic G3BP1 foci (Figure 4A). Next, we performed amperometric analysis of the isolated SGs. Following stimulation with arsenite and SG assembly, similar results were obtained from SGs formed inside these non-cancer cells with spikes appearing only from platinized CFME instead of CFME (Figure 4B). Thus, this demonstrates that both cancer and non-cancer cell lines form SG that contain ROS upon stimulation with arsenite. For the SGs of non-cancer cells, the half width of the $\mathrm{H}_{2} \mathrm{O}_{2}$ is $0.376 \mathrm{~ms}$, faster than that of SGs of cancer cells, indicating the existence of different structures of SGs between non-cancer and cancer cells as the release dynamics are closely related to the structure of SGs. The $N_{\text {molecules }}$ for the SGs of cancer and non-cancer cells does not differ significantly.

Both SGs and ROS play pivotal roles in redox regulation and cell signaling. Many signaling molecules associated with diseases can concentrate in SGs, with several shown to be regulated by SGs. The accumulation of the pro-apoptotic RACK1 results in the supression of MTK1-sPAK signaling and a blocking of the resulting apoptosis. ${ }^{[20]}$ Similarly, heat shock results in the assembly of SGs that trap TRAF2, impairing NF- $\kappa \mathrm{B}$ dependent proapoptotic and proinflammatory responses. ${ }^{[21]}$ Proinflammatory responses are also silenced by the sequestration within SGs of SRC-3, a regulator of several transcription factors promoting inflammation. ${ }^{[22]}$ These studies support a role for SGs as signaling hubs, and they are generally considered cytoprotective. ${ }^{[6,20,23]}$

$\mathrm{H}_{2} \mathrm{O}_{2}$ has been identified as a second messenger molecule in subcellular compartments. ${ }^{[2]}$ Nicotinamide adenine dinucleotide phosphate (NADPH) oxidase (Nox) is responsible for the generation of ROS in immune cells during phagocytosis. However, non-phagocytic Nox enzymes have been found to be involved in many subcellular locations such as the endoplasmic reticulum, nucleus, and mitochondria. ${ }^{[25]}$ One or more Nox isoforms could be located in several subcellular compartments within a single cell type. ${ }^{[26]}$ And, expression of Nox enzymes in subcellular domains can regulate their participation in varied signaling pathways. The influence of compartmentalized Nox in immune signaling pathways and the compartmentalization of $\mathrm{H}_{2} \mathrm{O}_{2}$ in survival signaling have been reported. ${ }^{[27]}$ As for SGs, these enzymes may be located in the more dynamic shell surrounding the SG cores and thus can rapidly exchange with the cytoplasm to provide rapid adaptation or response. Future studies will aim at characterizing the association of these ROS producing enzymes by microscopy to capture dynamic and transient association and a mass spectrometry analysis of their content in response to different stressors.

The localization of ROS in SGs reveals the role of SGs as signaling hubs, and these ROS might participate in SGsassociated physiological activities. While high concentration of $\mathrm{H}_{2} \mathrm{O}_{2}$ triggers SGs assembly, the presence of $\mathrm{H}_{2} \mathrm{O}_{2}$ within
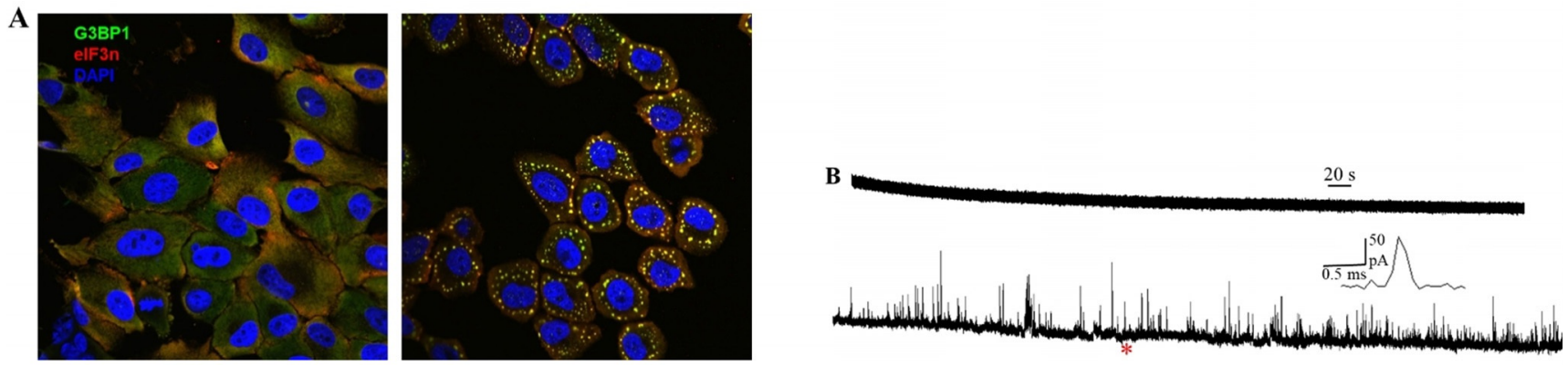

Figure 4. A) Representative fluorescence microscopy images of MCF-10A cells without (left) and with (right) $\mathrm{NaAsO}_{2}$ treatment (yellow dots represent SGs). B) Representative amperometric traces of SGs isolated from a non-cancer cell (MCF-10A) obtained from CFME (upper) and platinized CFME at $400 \mathrm{mV}$ vs. $\mathrm{Ag} / \mathrm{AgCl}$ (lower), the inset shows an amplification of the spike labeled with the red asterisk. 
SGs could be involved in feedback loop control of SG assembly by oxidizing essential proteins in the SGs, as previously shown for TIA1. This might sensitize the cells to a variety of pathological processes. ${ }^{[28]}$ Aging neuronal cells and tumor cells are more prone to encounter ER stress or oxidative stress. Consequently, ROS-mediated disturbance of SG formation could impair the cytoprotection provided by SGs and promote degeneration initiated by these SG-inducing as well as SG-non-inducing stresses.

In conclusion, using amperometry we have discovered that arsenite-induced SGs contain ROS in both cancer and non-cancer cells and that $\mathrm{H}_{2} \mathrm{O}_{2}$ is the main electroactive species in SGs. The structures of SGs between non-cancer and cancer cells are probably different as indicated by the different release dynamics. This significantly advances our understanding of SG composition beyond macromolecules such as RNA and proteins. The discovery of these small molecules will help uncover new functions for SGs associated with cellular physiology. These ROS might participate in SGsrelated activities including redox regulation and cell signaling. Yet, in some tumor cells and especially aging neuronal cells, ROS might impair SGs-mediated cytoprotection by oxidizing essential components of SGs and render the cells sensitive to pathological insults, which will promote neurodegeneration. Although the exact biological function of SGs remains poorly understood, this finding unveils a correlation between SG biology and pathogenesis in NDs and some cancers, which will offer new insights into the therapeutics of SG-related diseases. As ER stress and oxidative stress are implicated in other diseases such as diabetes, stroke and atherosclerosis, the malfunction of SGs formation might also be involved in these disorders.

Given the indication that the composition of SGs can differ significantly depending on cell type and stress stimuli, further investigation is needed to establish how SG heterogeneity impact on their capacity to store reactive species, and their impact on cellular function.

\section{Acknowledgements}

We acknowledge funding from the European Research Council (Advanced Grant NanoBioNext grant number 787534), the Knut and Alice Wallenberg Foundation, the Swedish Research Council (VR project number 2017-04366), and the Medical Research Council (MR/R02426X/1).

\section{Conflict of interest}

The authors declare no conflict of interest.

Keywords: amperometry - microelectrodes . neurodegeneration reactive oxygen species $\cdot$ stress granules

[1] J. R. Buchan, RNA Biol. 2014, 11, 1019-1030.

[2] H. Yoo, C. Triandafillou, D. A. Drummond, J. Biol. Chem. 2019, $294,7151-7159$.
[3] D. S. W. Protter, R. Parker, Trends Cell Biol. 2016, 26, 668 - 679.

[4] G. A. Corbet, R. Parker, Cold Spring Harbor Symp. Quant. Biol. 2019, 84, 203-215.

[5] C. L. Riggs, N. Kedersha, P. Ivanov, P. Anderson, J. Cell Sci. 2020, 133 , jes242487.

[6] N. Kedersha, P. Ivanov, P. Anderson, Trends Biochem. Sci. 2013, $38,494-506$.

[7] a) K. Begovich, A. Q. Vu, G. Yeo, J. E. Wilhelm, J. Cell Biol. 2020, 219, e201904141; b) M. Rollins, S. Huard, A. Morettin, J. Takuski, T. T. Pham, M. D. Fullerton, J. Côté, K. Baetz, PLoS Genet. 2017, 13, e1006626.

[8] N. Eiermann, K. Haneke, Z. Sun, G. Stoecklin, A. Ruggieri, Viruses 2020, 12, 984-1031.

[9] a) Y. R. Li, O. D. King, J. Shorter, A. D. Gitler, J. Cell Biol. 2013, 201, 361-372; b) M. Repici, M. Hassanjani, D. C. Maddison, P. Garção, S. Cimini, B. Patel, É. M. Szegö, K. R. Straatman, K. S. Lilley, T. Borsello, T. F. Outeiro, L. Panman, F. Giorgini, Mol. Neurobiol. 2019, 56, 61-77; c) T. Vanderweyde, H. Yu, M. Varnum, L. Liu-Yesucevitz, A. Citro, T. Ikezu, K. Duff, B. Wolozin, J. Neurosci. 2012, 32, 8270-8283.

[10] P. Anderson, N. Kedersha, P. Ivanov, Biochim. Biophys. Acta Gene Regul. Mech. 2015, 1849, 861-870.

[11] a) R. Mittler, Trends Plant Sci. 2017, 22, 11-19; b) B. D'Autréaux, M. B. Toledano, Nat. Rev. Mol. Cell Biol. 2007, 8, 813 824.

[12] D. R. Gough, T. G. Cotter, Cell Death Dis. 2011, 2, e213-8.

[13] a) M. T. Quinn, M. C. B. Ammons, F. R. DeLeo, Clin. Sci. 2006, 111,1-20; b) M. Valko, D. Leibfritz, J. Moncol, M. T. D. Cronin, M. Mazur, J. Telser, Int. J. Biochem. Cell Biol. 2007, 39, 44-84; c) F. R. DeLeo, L.-A. H. Allen, M. Apicella, W. M. Nauseef, J. Immunol. 1999, 163, 6732-6740.

[14] a) C. Amatore, S. Arbault, C. Bouton, K. Coffi, J. C. Drapier, H. Ghandour, Y. Tong, ChemBioChem 2006, 7, 653-661; b) K. Hu, Y. Li, S. A. Rotenberg, C. Amatore, M. V. Mirkin, J. Am. Chem. Soc. 2019, 141, 4564-4568.

[15] a) S. Jain, J. R. Wheeler, R. W. Walters, A. Agrawal, A. Barsic, R. Parker, Cell 2016, 164, 487-498; b) M. Brocard, V. Iadevaia, P. Klein, B. Hall, G. Lewis, J. Lu, J. Burke, M. M. Willcocks, R. Parker, I. G. Goodfellow, A. Ruggieri, N. Locker, PLoS Pathog. 2020, 16, e1008250.

[16] J. Dunevall, H. Fathali, N. Najafinobar, J. Lovric, J. Wigström, A. S. Cans, A. G. Ewing, J. Am. Chem. Soc. 2015, 137, 43444346.

[17] S. Arbault, P. Pantano, J. A. Jankowski, M. Vuillaume, C. Amatore, Anal. Chem. 1995, 67, 3382-3390.

[18] X. Li, L. Ren, J. Dunevall, D. Ye, H. S. White, M. A. Edwards, A. G. Ewing, ACS Nano 2018, 12, 3010-3019.

[19] Y. Li, K. Hu, Y. Yu, S. A. Rotenberg, C. Amatore, M. V. Mirkin, J. Am. Chem. Soc. 2017, 139, 13055-13062.

[20] K. Arimoto, H. Fukuda, S. Imajoh-Ohmi, H. Saito, M. Takekawa, Nat. Cell Biol. 2008, 10, 1324-1332.

[21] W. J. Kim, S. H. Back, V. Kim, I. Ryu, S. K. Jang, Mol. Cell. Biol. 2005, 25, 2450-2462.

[22] P. Anderson, N. Kedersha, Mol. Cell 2007, 25, 796-797.

[23] K. Thedieck, B. Holzwarth, M. T. Prentzell, C. Boehlke, K. Kläsener, S. Ruf, A. G. Sonntag, L. Maerz, S.-N. Grellscheid, E. Kremmer, R. Nitschke, E. W. Kuehn, J. W. Jonker, A. K. Groen, M. Reth, M. N. Hall, R. Baumeister, Cell 2013, 154, 859-874.

[24] T. Gill, A. D. Levine, J. Biol. Chem. 2013, 288, 26246-26255.

[25] a) K. Chen, M. T. Kirber, H. Xiao, Y. Yang, J. F. Keaney, Jr., J. Cell Biol. 2008, 181, 1129-1139; b) R.-M. Liu, J. Choi, J.-H. Wu, K. A. Gaston Pravia, K. M. Lewis, J. D. Brand, N. S. R. Mochel, D. M. Krzywanski, J. D. Lambeth, J. S. Hagood, H. J. Forman, V. J. Thannickal, E. M. Postlethwait, J. Biol. Chem. 2010, 285, $16239-16247$; c) K. A. Graham, M. Kulawiec, K. M. Owens, X. Li, M. M. Desouki, D. Chandra, K. K. Singh, Cancer Biol. Ther. 2010, 10, 223-231. 
[26] K. von Löhneysen, D. Noack, M. R. Wood, J. S. Friedman, U. G. Knaus, Mol. Cell. Biol. 2010, 30, $961-975$.

[27] a) M. Ushio-Fukai, Antioxid. Redox Signaling 2008, 11, 1289 1299; b) S. Lipinski, A. Till, C. Sina, A. Arlt, H. Grasberger, S. Schreiber, P. Rosenstiel, J. Cell Sci. 2009, 122, 3522-3530; c) R.F. Wu, Z. Ma, Z. Liu, L. S. Terada, Mol. Cell. Biol. 2010, 30, $3553-3568$.
[28] K. Arimoto-Matsuzaki, H. Saito, M. Takekawa, Nat. Commun. 2016, 7, 1-10.

Manuscript received: March 28, 2021

Revised manuscript received: April 15, 2021

Accepted manuscript online: April 19, 2021

Version of record online: June 9, 2021 